\title{
How Far Could these New SARS-CoV-2 Variants Take Us?
}

\section{Fabiana Avila Carneiro ${ }^{1,2 *}$ and Lucio Ayres Caldas ${ }^{1,2}$}

${ }^{1}$ Núcleo Multidisciplinar de Pesquisas em Biologia - NUMPEX-BIO, Campus Duque de Caxias Geraldo Cidade, Universidade Federal do Rio de Janeiro, Rio de Janeiro, Duque de Caxias, RJ, Brazil

${ }^{2}$ Laboratório de Ultraestrutura Celular Hertha Meyer, Instituto de Biofísica Carlos Chagas Filho, Universidade Federal do Rio de Janeiro, Brazil
Received: April 14, 2021

Published: May 17, 2021

(C) All rights are reserved by Fabiana Avila Carneiro and Lucio Ayres Caldas.

*Corresponding Author: Fabiana Avila Carneiro, Núcleo Multidisciplinar de Pesquisa em Biologia - NUMPEX-BIO, Campus Duque de Caxias Geraldo Cidade, Universidade Federal do Rio de Janeiro, Rio de Janeiro, Duque de Caxias, RJ, Brazil. Email: fcarneiro@caxias.ufrj.br

COVID-19 first cases emerged in Wuhan, China, spreading across other countries, culminating in a global pandemic [1]. Regarding the analysis of the clinical characteristics of patients infected with the new coronavirus, although all ages are susceptible, the published studies showed an average age between 40 and 56 years and a higher incidence in men, but it is clear that more serious cases are related to older population and individuals with associated diseases (obesity, diabetes, heart disease) [2]. COVID-19 in some cases can progress to pneumonia, acute respiratory distress syndrome (ARDS) and multiple organ dysfunction [3].

SARS-CoV-2 causes a severe pneumonia, which differentiates COVID-19 from a mild flu and because of that, it was possible to observe the entry of this new pathogen [4]. From then on, isolation, sequencing and identification through molecular tests were necessary, which were made available by the networks so that all scientists had access to this data.

An important movement to detect new variants was the genome sequence. By sequencing the samples, an "open reading frame" (ORF8) was observed that would be phylogenetically close to another coronavirus called SARS-CoV, but in a different strain [5]. In addition, the amino acid sequence of the "receptor-binding domain" (RBD) also has similarities to that of SARS-CoV, which is known to have originated from bats. These findings highlight the importance and urgent need for regular surveillance of interspecies transmission, as of $\mathrm{CoV}$ from bats to humans [6].

During the progress of pandemia, new variants of SARS-CoV-2 have arised, causing varying degrees of concern because some are associated with increased infectivity and lethality [7]. It is certainly worrying to expand due to such diversity, which leads us to fear that we will never achieve collective immunity. However, there is growing evidence that these variants share similar combinations of mutations, suggesting that there may be a finite number of new viral versions.

The first mutation at SARS-CoV-2 spike protein observed was S: $614 \mathrm{G}$, which was identified and spread rapidly, being found in 90\% of infections today. The line B.1.525 shows variation $\mathrm{Q} 677 \mathrm{H}$, it is currently being found in the USA, Egypt, Denmark, India and part of Macedonia, being a descendant of line B.1.1.7, the most worrying version at the moment due to the alleged in the efficiency of contagion. The appearance and gain in prevalence of the B.1.525 mutation suggests that it improves replication in some way not yet detected, as it is found in a region of protein S, responsible for the interaction and entry of the virus into the human cell. Mutations in at least eight different positions in the spike protein are simultaneously on the rise worldwide, appearing in B.1.1.7 and in other important variants known as B.1.351, P.1 and P.3. These variants share combinations of mutations at positions $18,69-70,417,452$, 501, 681 and a particularly important E484K mutation, as it prevents the production of neutralizing antibodies [8].

Variant B.1.1.7 was identified in the United Kingdom with a large number of mutations in the fall of 2020 [9]. Although this variant was announced as a variant that spreads more easily and quickly, this has not yet been demonstrated. In January 2021, this variant was reported to be associated with an increased risk of death compared to other variants of the virus, but further studies are needed to confirm this finding. Since then, it has been detected in many countries around the world [10].

In South Africa, another variant called B.1.351 [11] emerged independently of B.1.1.7. Originally detected in early October 2020, B.1.351 shares some mutations with B.1.1.7. 
In Brazil, a variant called P.1 emerged and was first identified in travelers from Brazil, who were tested during routine screening at an airport in Japan in early January 2021. This variant contains a set of additional mutations that can affect its ability to be recognized by antibodies.

It is important to note that the fact that a variant was detected in a given country, does not mean that this variant necessarily arised there. The history of the great epidemics has taught us that. The "Spanish Flu" was not spanish, HIV was first isolated in Los Angeles, but now we know that the virus arised in West-central Africa [12]. In view of this, we can not rule out the first emergence of SARS-CoV-2 in other country than China. This is, besides, a good reason to avoid officially referring to the variants together with the country where it was firstly detected.

Since many newly discovered variants appear to be rearranging the mutations found in other established variants, we can speculate that the virus is beginning to run out of new and important adaptations. But that does not mean that the forces of evolution will stop when we start to approach collective immunity and loosen restrictions. It is known that the quasispecies infection can lead to a founder effect that results in the adaptation of some variants that will, consequently, spread widely. Furthermore, the selective pressures during the virus interaction with all kinds of immunological conditions may eventually result in a new strain, i.e. a virus with significant and stable phenotypic alterations, capable of escaping the barriers that prevent transmission. This is especially possible when infections remain numerous. We must remember that the more infections there are, the greater the chance of mutations occurring, and those that better help the virus survive will proliferate. That is why preventing new infections is essential.

\section{Bibliography}

1. Zhu N., et al. "A Novel Coronavirus from Patients with Pneumonia in China, 2019". The New England Journal of Medicine 382 (2020): 727-733.

2. Li F, et al. "Household transmission of SARS-CoV-2 and risk factors for susceptibility and infectivity in Wuhan: a retrospective observational study". Lancet Infectious Diseases 20 (2021): 30981-30986.

3. Zaim S., et al. "COVID-19 and Multiorgan Response". Current Problems in Cardiology 45.8 (2020):100618-100640.

4. Lauxmann M., et al. "The SARS-CoV-2 Coronavirus and the COVID-19 Outbreak". International Brazilian Journal of Urology 46 (2020): 6-18.
5. Russell Y., et al. "Atypical Divergence of SARS-CoV-2 Orf8 from Orf7a within the Coronavirus Lineage Suggests Potential Stealthy Viral Strategies in Immune Evasion". mBio Clinical Science and Epidemiology 12.1 (2021):e03014-e03020.

6. Li-Li Ren., et al. "Identification of a novel coronavirus causing severe pneumonia in human: a descriptive study". Chinese Medical Journal (Engl) 133.9 (2020): 1015-1024.

7. World Health Organization. "WHO Coronavirus Disease (COVID-19) Dashboard" (2020).

8. Hoffmann M., et al. "SARS-CoV-2 variants B.1.351 and P.1 escape from neutralizing antibodies". Cell 184.9 (2021): 23842393.e12.

9. Pan D., et al. "The new UK SARS-CoV-2 variant and lockdown - causes and consequences". Clinical Medicine (Lond) (2021):clinmed.0019.

10. Lauring AS., et al. "Genetic Variants of SARS-CoV-2-What Do They Mean?". JAMA 325.6 (2021):529-531.

11. Luan B., et al. "Insights into SARS-CoV-2's Mutations for Evading Human Antibodies: Sacrifice and Survival". Journal of Medicinal Chemistry (2021).

12. Giovanetti M., et al. "Molecular Epidemiology of HIV-1 in African Countries: A Comprehensive Overview". Pathogens 9.12 (2020):1072.

Volume 4 Issue 6 June 2021

C All rights are reserved by Fabiana Avila Carneiro and Lucio Ayres Caldas. 